\title{
SINGAPOREAN HEALTH CARE PROFESSIONALS' KNOWLEDGE AND OPINION ABOUT MEDICATION USE IN BREASTFEEDING
}

\author{
Y.Ng \\ Neonatology, National University Hospital, Singapore, Singapore
}

Background: Medication is frequently prescribed for post-partum mothers. Inappropriate advice by health care professionals may result in low medication compliance or disruption of breastfeeding. This survey aimed to assess knowledge and opinion of a select group of health care professionals.

Methods: Attendees of a seminar "How to manage problems of the mother-child in the first month" were invited to complete a pre-lecture survey on "Breastfeeding and medications". Ten statements were answered true, false or don't know: Eight statements assessed factual knowledge, 2 assessed attendees' opinion.

Results: Sixty-five people responded: 38 nurses, 8 general practitioners, 7 lactation consultants, remaining 12 did not specify their profession. For the 8 factual questions, only $35 \%$ were correctly answered, $53 \%$ were wrong, $12 \%$ answered "don't know". Lactation consultants fared best, answering $60 \%$ of statements correctly, followed by doctors $(46 \%)$ and nurses $(30 \%)$. About half the participants correctly answered that mothers receiving antibiotics for mastitis can continue breastfeeding, and propylthiouracil is compatible with breastfeeding. However, nearly two-thirds wrongly perceived drug directories as good references for breastfeeding compatible drugs. Many participants (41\%) would advise mothers to stop breastfeeding if effects of drugs on the infant were of a concern.

Conclusions: Knowledge about effects of medication during breastfeeding is low amongst health professionals who have close contact with the mother and newborn. This situation could result in inappropriate breastfeeding advice unless efforts are made to bridge these gaps in knowledge.

Acknowledgement: Association for Breastfeeding Advocacy (Singapore) and survey responders 